\title{
CORRIGENDUM / RECTIFICATIF
}

\section{Corrigendum: Examination of the Clinical Queries and Systematic Review 'Hedges' in EMBASE and MEDLINE}

\section{Susan M. Bradley}

Ref. JCHLA, 2010; 31(2):27-37. DOI 10.5596/c10-022

In Table 2, row 1, the EMBASE search string for the hedge 'Diagnosis (2 or more terms min difference)' was incorrectly listed as 'sensitive*.tw. OR diagnostic accuracy.sh. OR diagnostic.tw.'

The search as run was 'sensitiv:.tw. OR diagnostic accuracy.sh. OR diagnostic.tw.'
In Table 2, row 2, the EMBASE search string for the hedge 'Reviews ( 2 or more terms, best optimization)' was incorrectly listed as "meta-analysis*.mp. OR search*.tw. OR review.pt.'

The search as run was 'meta-analys:.mp. OR search:.tw. OR review.pt.' 